\title{
Dectin-2 sensing of house dust mite is critical for the initiation of airway inflammation
}

\author{
DL Clarke ${ }^{1}$, NHE Davis ${ }^{1}$, CL Campion ${ }^{1}$, ML Foster $^{2}$, SC Heasman ${ }^{3}$, AR Lewis ${ }^{3}$, IK Anderson ${ }^{1,4}$, \\ DJ Corkill ${ }^{1}$, MA Sleeman ${ }^{1}, \mathrm{RD} \mathrm{May}^{1}$ and MJ Robinson ${ }^{1}$
}

How the immune system senses aeroallergens and triggers an aberrant inflammation is poorly understood. Dectin-2 is a house dust mite (HDM)-sensing pattern recognition receptor. In a 3-week mouse model of repeated intranasal HDM challenge, anti-Dectin-2 potently attenuated the characteristic allergic inflammation and airway hyper-responsiveness. Anti-Dectin-2 also prevented neutrophil influx following a single HDM challenge. Interestingly, cysteinyl leukotrienes, but not chemokine and cytokine levels were inhibited by anti-Dectin-2 in this acute model, and in ex vivo challenge of cultured alveolar macrophages with HDM. Furthermore in the single-challenge model, zileuton, an inhibitor of leukotriene production, produced a similar effect as Dectin-2 blockade. Together these data suggest alveolar macrophage sensing of HDM by Dectin-2 elicits the production of cysteinyl leukotrienes, and this axis is key for the initiation of airway inflammation to this aeroallergen. Finally, we found Dectin-2-positive infiltrating cells present in bronchial biopsies from asthmatic subjects.

\section{INTRODUCTION}

Asthma is a complex and heterogeneous disease yet the airway inflammation that underlies the disease tends to be allergic in nature. The majority of asthmatic subjects are sensitive to one or more allergens, and indeed studies show that 50-95\% patients are sensitive to the prototypic aeroallergen house dust mite (HDM). ${ }^{1}$ What triggers a pathogenic allergic response to seemingly innocuous substances is poorly understood. Pattern recognition receptors (PRRs), expressed by innate immune cells, have a fundamental role in the initial sensing of microbes and instructing an appropriate inflammatory and adaptive response. ${ }^{2}$ Therefore, it has been proposed that allergens anomalously engage PRRs, thereby provoking inflammation and Th2 immunity.

HDM has been extensively studied ex vivo and in the mouse lung. HDM is able to agonize many PRRs including: formyl peptide receptor (FPR) and FPR-like 1 on eosinophils; ${ }^{3}$ PAR-2 on epithelial cells; ${ }^{4}$ TLR4 on stromal cells ${ }^{5}$ and Dectin-2 on dendritic cells. ${ }^{6}$ Significantly, TLR4 and Dectin-2 have been shown to be required in in vivo models of airway inflammation. ${ }^{5,7}$ Stromal TLR4, presumably on epithelial cells, is absolutely required for HDM-induced airway inflammation yet leukocyte TLR4 is not engaged or is redundant. ${ }^{5}$ Dendritic cell Dectin-2 expression is required for instructing a Th2skewed adaptive response, as discussed below. ${ }^{7}$ So what is apparent is the induction of an allergic response to a complex aeroallergen such as HDM is due to more than one PRR on more than one cell type.

The only PRR with a clearly defined role in innate immune cell activation induced by HDM is the myeloid C-type lectin Dectin-2. ${ }^{7}$ Antibody-mediated clustering of Dectin-2 on bone marrow-derived dendritic cells leads to cytokine induction, yet on the same cell type the receptor is partially redundant for the induction of cytokines by HDM or its other ligand fungi. ${ }^{7,8}$ Despite this, Dectin-2 is necessary for instructing a Th2 response to HDM due to the induction of cysteinyl leukotrienes from dendritic cells. ${ }^{7}$ Interestingly Dectin-2 is critical for the Th17 immunity to fungi. ${ }^{8}$ In the lungs of naïve mice, Dectin-2 is expressed primarily on $\mathrm{CD}^{+} 8^{+} \mathrm{CD} 11 \mathrm{c}^{\text {low }}$ cells likely to be alveolar macrophages, ${ }^{9}$ suggesting its contribution to HDMdriven airway inflammation may not be restricted to instructing the adaptive response.

\footnotetext{
${ }^{1}$ Departments of Respiratory, Inflammation and Autoimmunity, Medlmmune Ltd, Cambridge, UK. ${ }^{2}$ Department of Pathology, Safety Assessment UK, AstraZeneca R\&D, Loughborough, UK. ${ }^{3}$ Research Histology, Medlmmune Ltd, Cambridge, UK and ${ }^{4}$ Present address: Johnson \& Johnson, London, UK. Correspondence: MJ Robinson (robinsonm@medimmune.com)
} 
We sought to investigate the role of Dectin-2 in the initiation and maintenance of airway inflammation and found that Dectin-2 is critical for induction of HDM-mediated airway inflammation, an effect mimicked by the leukotriene inhibitor zileuton. In vivo and ex vivo experiments with alveolar macrophages confirmed a key role for Dectin-2 in the induction of cysteinyl leukotriene release triggered by HDM. In addition, we also demonstrate the expression of Dectin-2 in the airways of patients with asthma.

\section{RESULTS}

\section{Dectin-2 is required for HDM-induced airway} hyper-responsiveness (AHR) and inflammation

To fully understand the role of Dectin-2 in an allergic response to HDM in the lungs, we used a chronic 3-week HDM model. To neutralize Dectin-2, one group was treated $24 \mathrm{~h}$ before the first HDM dose and twice weekly thereafter with the blocking antibody, D2.11E4. ${ }^{8}$ Anti-Dectin-2, but not isotype control antibody, prevented the HDM-induced increase in lung resistance (Figure 1a) and elastance (Supplementary Figure S1A online) in response to methacholine challenge. The effect was similar to the positive control prednisone. This indicates Dectin-2 activation is critical for development of HDM-driven allergic AHR.

The HDM-driven inflammation in the lung was also dramatically reduced and altered in nature by the blocking of Dectin-2. Anti-Dectin-2 treatment reduced the total cell counts in BALF (bronchoalveolar lavage fluid) and recruitment of eosinophils and neutrophils, as potently as corticosteroid treatment (Figure 1b-d). Anti-Dectin-2 did not have a significant effect on monocyte/macrophage and lymphocyte numbers (data not shown). These data were reflected in an apparent reduction in inflammation in the lungs as determined by histology (Figure 1e-j).

Repeated HDM challenge also resulted in an increase in several cytokines and chemokines in the lung. Consistent with the decreased neutrophil and eosinophil infiltrate, Dectin-2 blockade reduced the level of the neutrophil and eosinophil chemoattractants CXCL1/KC, CCL11/Eotaxin and CCL5/ RANTES (Figure 1k,l, Supplementary Figure S1E). The pro-cytokines IL-1 $\beta$, as well as the more anti-inflammatory IL-10, were also attenuated (Supplementary Figure S1B,D). The Th2 cytokines, IL-4 and IL-5, were also significantly reduced (Figure 1m, Supplementary Figure S1C), supporting a reduced adaptive response observed by Barrett et al." However, this could also in part reflect a decrease in CCR4 chemokines CCL22/MDC and CCL17/TARC (Supplementary Figure S1F,G). Interestingly of all the induced cytokines and chemokines, only the pro-Th1/17 cytokine IL-12p40 (total IL-12 and IL-23) was not impacted by anti-Dectin-2 treatment (data not shown). Cysteinyl leukotriene levels were not significantly raised at this time point (data not shown). Therefore, Dectin-2 blockade in the chronic HDM model did not prevent the global inflammatory response, but did lead to a down modulation of Th2 cytokines and a decreased chemokine signature. In summary, these data clearly demonstrate a non-redundant role for Dectin-2 in the development of HDMdriven allergic airway inflammation and lung pathophysiology.

\section{Dectin-2 is not required in an established HDM-driven inflammation}

Prophylactic blockade of Dectin-2 demonstrated a nonredundant role in the development of an allergic response to pulmonary challenge with HDM. Next, we addressed the role of Dectin-2 in established inflammation. In contrast to dosing anti-Dectin-2 throughout the chronic challenge, blocking Dectin-2 for the final 2 weeks of the 3-week model had little impact on the resultant AHR (Figure 2a, Supplementary Figure S2A), airway inflammation as determined by cellular infiltrate (Figures $\mathbf{2 b}$-d) or chemokine and cytokine levels in the lung (Supplementary Figure S2B-J). These data suggest that Dectin-2 is either largely redundant or has no role in an established allergic inflammation.

\section{Dectin-2 is essential for the initial response to HDM}

The efficacy of prophylactic, but not therapeutic, Dectin-2 blockade in attenuating the AHR and inflammation observed in a chronic HDM model led us to hypothesize that Dectin-2 could be involved in the initiation of inflammation. To assess this, we developed an acute 24-h HDM model where BALB/c mice were given a single intranasal challenge with $25 \mu \mathrm{g}$ of $\mathrm{HDM}$ extract. At $24 \mathrm{~h}$ following the challenge, we observed a mild neutrophillic infiltrate in the BALF (Figure 3a) but no increase in monocyte/macrophages, eosinophils or lymphocytes (data not shown). CXCL1/KC, TNF $\alpha$ (Figure 3b,c), and IL-12p40 (Supplementary Figure S3A) were also significantly elevated in the BALF. However, the Th2 cytokines, IL-4 and IL-5, as well as IL-2, IL-10, and IL-1 $\beta$ were not robustly induced in the model (data not shown). At the mRNA level, we did observe a greater than twofold increase in IL-1 $\beta$, IL-12p40, CCL2/JE, CCL3/MIP1 $\alpha$, CCL22/MDC, CXCL1/KC, CXCL2/MIP2, and CXCL9/MIG (Supplementary Figure S3B-I) but none of the other chemokines and cytokines measured (Supplementary Table S1). We did not observe any increase in AHR in similar acute models (data not shown).

Next, we evaluated the contribution of Dectin-2 in this single HDM challenge model by dosing with anti-Dectin- 2 the day before HDM challenge. Anti-Dectin-2, but not the isotype control, potently inhibited the neutrophilia by $77 \%$ (Figure 3a). However, the levels of CXCL1/KC, TNF $\alpha$, and IL-12p40 were largely unaffected (Figures 3b,c, Supplementary Figure S3A). Additionally, the cytokines and chemokines that were raised at the mRNA level were also not affected significantly by Dectin- 2 blockade (Supplementary Figure S3B-I). Therefore, although anti-Dectin-2 blocks HDM-triggered neutrophil infiltration, this did not appear to be modulating the induction of cytokines and chemokines.

HDM triggering of Dectin-2 can lead to the production of cysteinyl leukotrienes by dendritic cells. ${ }^{6}$ In the acute HDM challenge, cysteinyl leukotrienes were transiently induced with a peak at $2 \mathrm{~h}$ (data not shown), and had returned to baseline at $24 \mathrm{~h}$. The peak cysteinyl leukotriene levels at $2 \mathrm{~h}$ was blocked by 

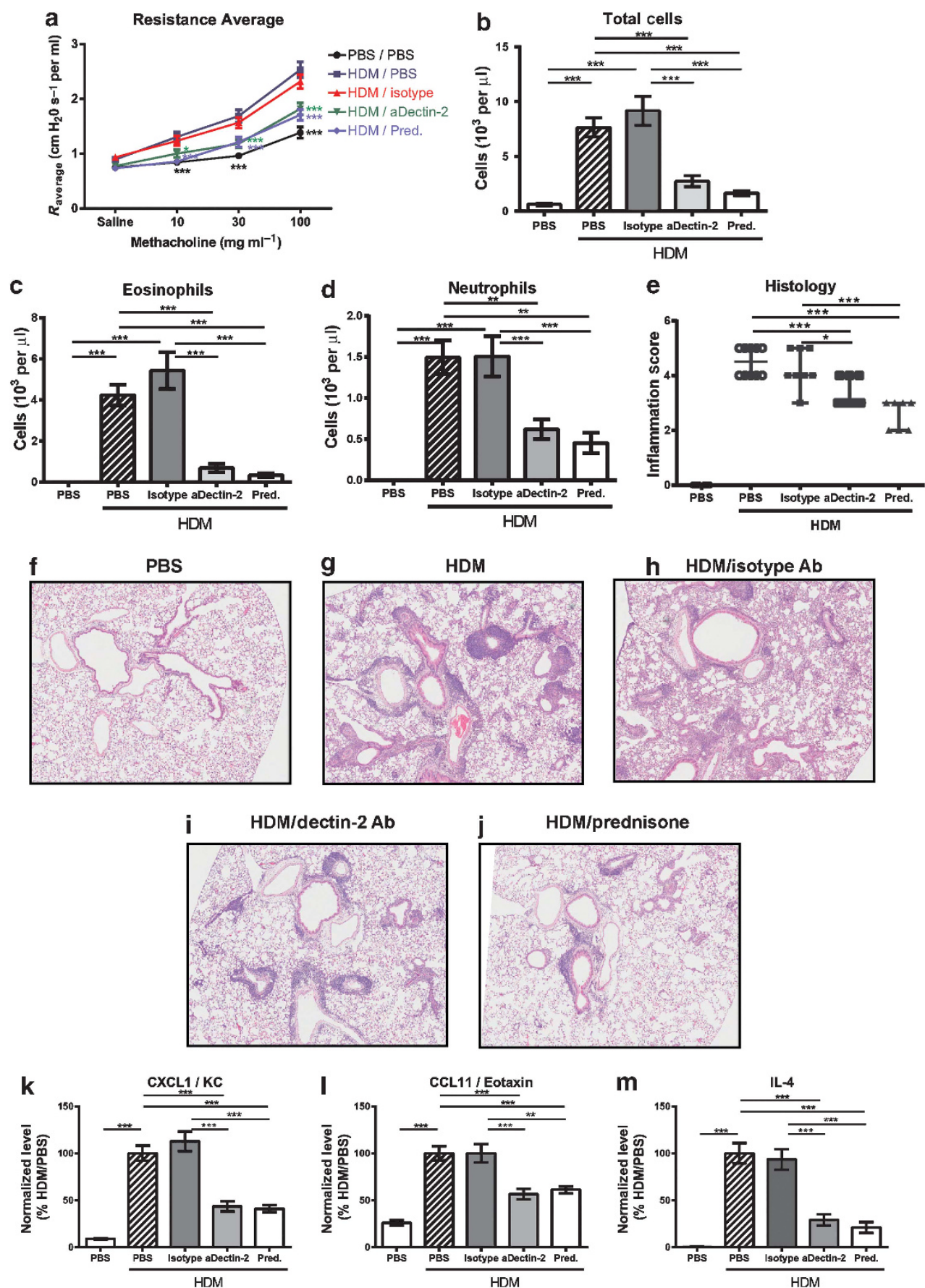

Figure 1 Neutralisation of Dectin-2 before house dust mite (HDM) allergen challenge ablates airway inflammation and airway hyper-responsiveness (AHR). Mice were treated with phosphate-buffered saline (PBS), anti-Dectin-2 or isotype control 1 day before and throughout the 3-week chronic HDM model or with prednisone for the final 2 weeks. Total airways resistance was determined in response to increasing concentrations of methacholine, as a measure of AHR (a). Cellular recruitment to the airway lumen was determined by bronchoalveolar lavage, followed by differential counting of total cells (b), eosinophils (c) and neutrophils (d). Histology was performed using hematoxylin and eosin staining on formalin-fixed lung parenchyma (f-j; $\mathrm{bar}=500 \mu \mathrm{m})$, and assessed by a semi-quantitative scoring system (0-5) (e). Lungs were homogenized and assayed for cytokine content by Meso-Scale Discovery or enzyme-linked immunosorbent assay. Levels were normalized to mean values from HDM/PBS-treated animals for each independent experiment $(\mathbf{k}-\mathbf{m})$. With the exception of e (median \pm s.e.m.; range $n=6-10)$, data are expressed as mean \pm s.e.m., $n=18-30$ mice per group and are a composite of three independent experiments. Significance was determined by 1-way analysis of variance (ANOVA) comparing all groups, but from untreated animals to all groups not shown. For a, 2-way ANOVA was used, and significance to HDM/PBS shown. ${ }^{*} P<0.05,{ }^{* \star} P<0.01,{ }^{* *} P<0.001$.

treatment with Dectin-2 antibody (Figure 3d). At this time point, the BALF cells counts were the same as naïve and HDMonly treated animals, and consisted of $>96 \%$ monocyte/ macrophages with infiltrating neutrophils accounting for less than $3 \%$ (data not shown). In the lungs of naïve mice, Dectin-2 is expressed primarily on alveolar macrophages, ${ }^{9}$ and by FACS, 

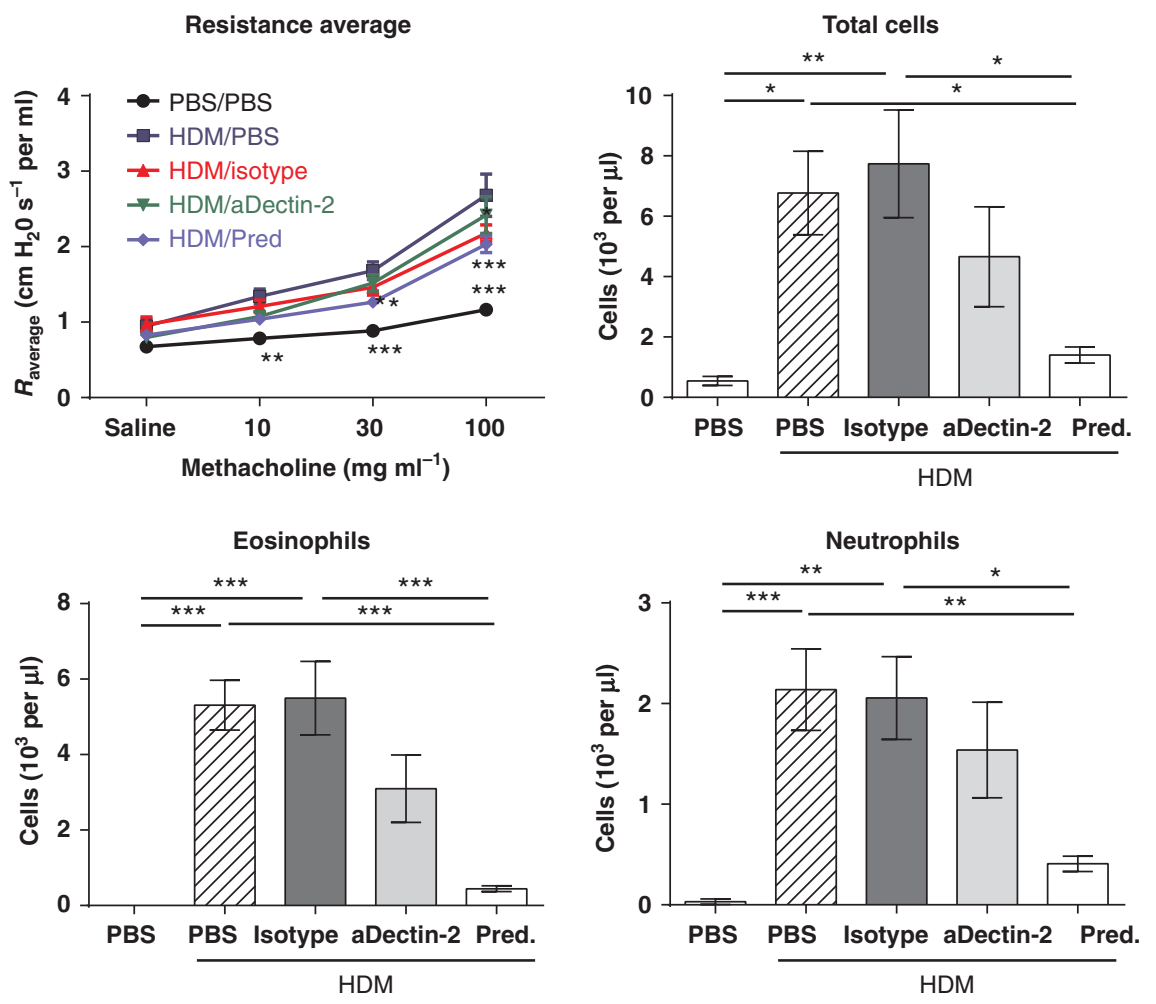

Figure 2 Dectin-2 is redundant in established allergic inflammation induced by house dust mite (HDM). Mice were sensitized and challenged as per the HDM chronic model with anti-Dectin-2 or isotype control antibody dosed only during the final 2 weeks. Total airways resistance was determined in response to increasing concentrations of methacholine, as a measure of airway hyper-responsiveness (a). Cellular recruitment to the airway lumen was determined by bronchoalveolar lavage, followed by differential counting of total cells (b), eosinophils (c) and neutrophils (d). Data are expressed as mean \pm s.e.m., $n=18-30$ mice per group and are a composite of three independent experiments. Significance was determined by 1-way analysis of variance (ANOVA) comparing all groups except a, where a 2-way ANOVA was used and only significance to HDM/PBS shown. ${ }^{\star} P<0.05,{ }^{\star \star} P<0.01,{ }^{\star * \star} P<0.001$.

F4/80-positive macrophages in the BALF from unchallenged mice expressed Dectin-2 (data not shown). This indicates the key source of HDM-induced cysteinyl leukotrienes is likely to be alveolar macrophages.

\section{Dectin-2 is required for HDM-triggered cysteinyl leukotriene release from alveolar macrophages}

To demonstrate that Dectin-2 on alveolar macrophages can induce cysteinyl leukotriene production, we stimulated cultured GM-CSF-primed BALF cells ex vivo with HDM. Typically, these cultures contained $>97 \%$ alveolar macrophages, as defined by F4/80 staining. The GM-CSF priming was to reproduce the conditions within the lung where it is constitutively expressed (data not shown and Cates et al. ${ }^{10}$ ), and enhance the induction of cysteinyl leukotrienes (data not shown and Suram et al. ${ }^{11}$ ). The release of cysteinyl leukotrienes could be detected as soon as 5 min following stimulation with $10 \mu \mathrm{g} \mathrm{ml}^{-1} \mathrm{HDM}$ (data not shown) but was maximal at $30 \mathrm{~min}$. This response was potently inhibited by anti-Dectin-2, but not an isotype control (Figure 4a). These data indicate that the sensing of HDM by Dectin-2 on alveolar macrophages triggers cysteinyl leukotriene production as previously observed in GMCSF-differentiated dendritic cells. ${ }^{6}$

After $24 \mathrm{~h}$ of stimulation CXCL1/KC, TNF $\alpha$, and IL-10 were also produced by the BALF cells (Figure $4 \mathbf{b}-\mathbf{d}$ ), but IL-12p40,
IL-4, IL-5, IFN $\gamma$, IL- $1 \beta$ and IL- 2 were not raised or could not be detected (data not shown). In the BALF cells, the mRNA for CXCL1/KC, CXCL2/JE, CCL3/MIP1 $\alpha$, IL-1 $\alpha$, IL-1 $\beta$, CCL5/ RANTES and IL-6, but no other measured gene (Supplementary Table S1), was also increased by over twofold $4 \mathrm{~h}$ following HDM stimulation (Supplementary Figure S4A-G). Interestingly, the levels of none of the cytokines or chemokines were appreciably affected by Dectin-2 blockade at either the protein or mRNA level (Figure $\mathbf{4 b}$-d and Supplementary Figure S4A-G, respectively). Together, this suggests that Dectin-2 on alveolar macrophages has a non-redundant role for the production of cysteinyl leukotrienes but not cytokines and chemokines.

To strengthen our hypothesis that Dectin-2-induced cysteinyl leukotriene production is instrumental in the initiation of HDM inflammation, we determined the effect of zileuton, an inhibitor of 5-lipoxygenase, and therefore of leukotriene production, in the single-challenge HDM model. Like Dectin-2 blockade, zileuton potently attenuated the neutrophil infiltration but not the induction of cytokines and chemokines at the protein or mRNA level (Figure 5a-c and Supplementary Figure S5A-H). We also dosed zileuton throughout the chronic HDM model to support a role for cysteinyl leukotrienes in driving this response. Zileuton $\left(10\right.$ and $50 \mathrm{mg} \mathrm{kg}^{-1}$ p.o. twice daily) led to a reduction in HDM-induced BALF 

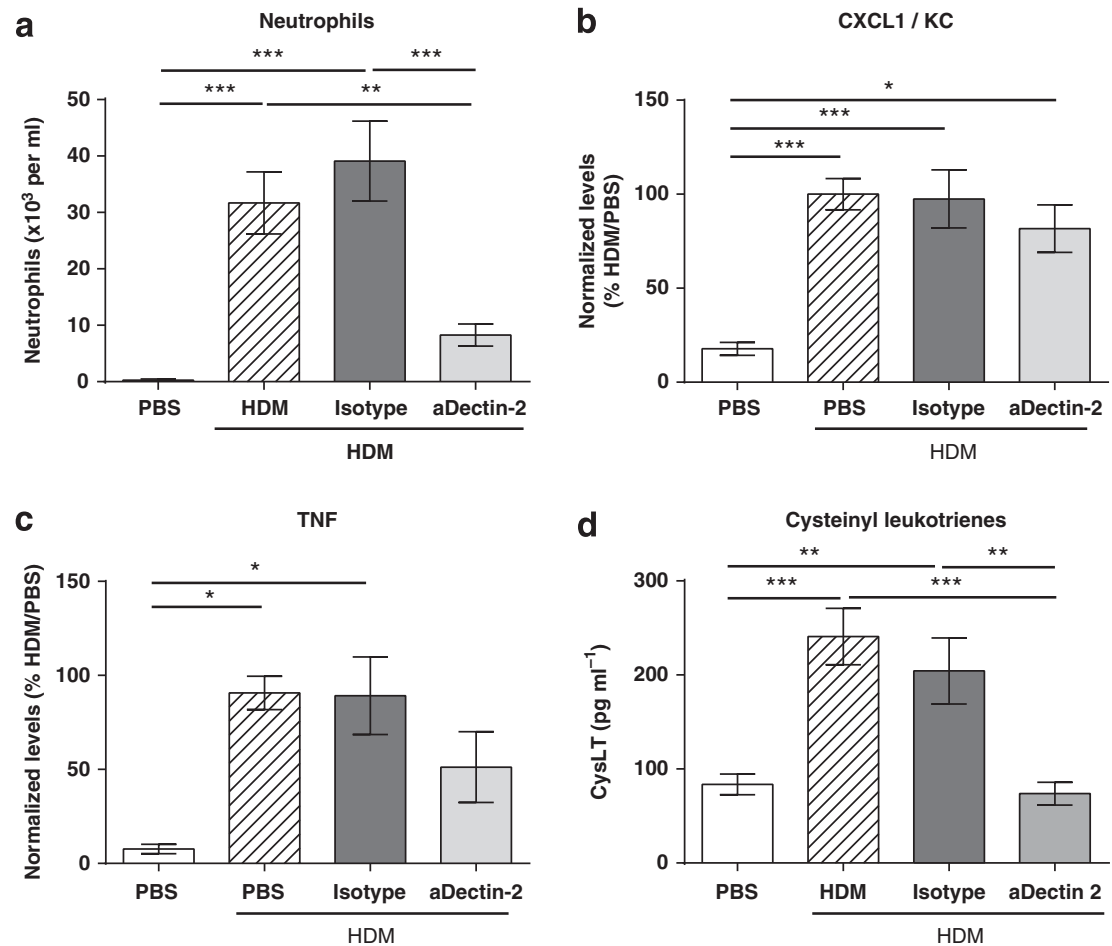

Figure 3 Neutrophil recruitment, but not cytokine or chemokine production, is Dectin-2 dependent in response to a single house dust mite (HDM) challenge. Mice were treated with anti-Dectin-2 or isotype control 1 day before a single HDM challenge. Cellular recruitment to the airway lumen was determined by bronchoalveolar lavage, followed by differential counting $24 \mathrm{~h}$ following challenge (a). BALF was assayed for cytokine content by Meso-Scale Discovery, and levels were normalized to mean values from HDM/PBS-treated animals for each independent experiment (b-c). (d) Cysteinyl leuokitrene levels were determined by in BALF $2 \mathrm{~h}$ following HDM challenge. Data are expressed as mean $\pm \mathrm{s}$.e.m., $n=7-12 \mathrm{mice}$ per group and are a composite of two independent experiments. Significance was determined by 1-way analysis of variance comparing all groups. ${ }^{\star} P<0.05,{ }^{* \star} P<0.01,{ }^{\star * \star} P<0.001$.

eosinophilia, as well as HDM-induced AHR (Supplementary Figure S6A,B).

Taken together the ex vivo, acute and chronic HDM challenge model data indicate the requirement for Dectin-2 in HDM-driven lung inflammation which is through the induction of cysteinyl leukotrienes.

\section{Expression of Dectin-2 in normal and asthmatic lung}

Immunohistochemical analysis of Dectin-2 in the bronchial biopsies from 12 GINA 1-4 asthmatic patients showed staining of some infiltrating leukocytes throughout the lamina propria (Figure 6b; see Supplementary Table S2 for demographic data). Because of the relatively low number of samples, it was not considered robust to make an analysis in relation to GINA staging. There was however no striking difference in Dectin-2 expression across disease severity. Quantification of the Dectin2-positive cells per area of lamina propria (Figure 6e) or length of epithelium (data not shown) did not reveal an increase above healthy normal controls biopsies (Figure 6a). On the basis of cytological morphology (comparing Dectin-2-stained slides with parallel haematoxylin and eosin sections), the positive cells were predominately mononuclear and occasional macrophage forms with neutrophils being consistently negative. This staining was not observed with a control antibody $\left(<0.0002\right.$ cells $\mu \mathrm{m}^{-2}$; Figure $\left.\mathbf{6 c}, \mathbf{d}\right)$. Therefore, Dectin-2 is expressed by infiltrating cells in the lung tissue of asthmatic patients, so it could contribute to airway inflammation triggered by HDM. However, the similar expression levels in healthy normals suggests it is unlikely to be the cause of increased aeroallergen sensitivity.

\section{DISCUSSION}

How aeroallergens are sensed by the innate immune system and trigger a pathological lung inflammation is a key mechanism in asthma, but is poorly understood. Here we show that blockade of Dectin-2 prevented airway inflammation and AHR in a chronic HDM model. This is consistent with the reported reduction in cellular infiltrate observed in a sensitization and challenge HDM model. ${ }^{7}$ We noted a more pronounced reduction in inflammation than Barrett et al. ${ }^{7}$ possibly due to the higher and continual dosing of anti-Dectin-2, the difference in HDM species, and/or variations in the HDMdosing regimens. Therapeutic dosing was ineffectual, suggesting a non-redundant role for Dectin-2 in the initiation, but not the maintenance, of inflammation. Using an acute single HDM challenge model, we demonstrated a critical role for Dectin-2 in the immediate inflammatory response to aeroallergen. Dectin2 has previously been shown to be critical for dendritic cells to instruct a Th2 skewed immunity to $\mathrm{HDM}^{7}$ However, the immediate inflammation we observe in the acute model is presumably independent of the adaptive response, and thus this dendritic cell-mediated mechanism. Additionally, 
a

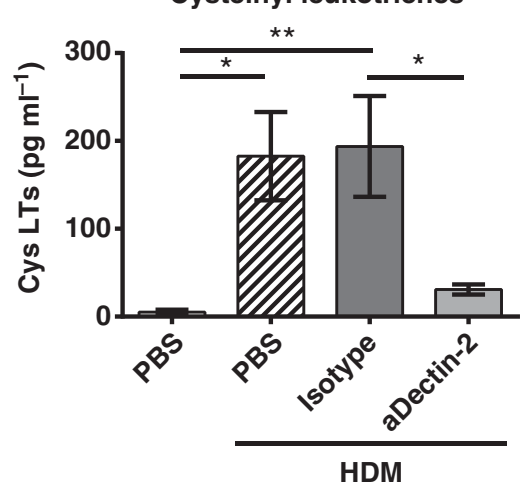

C

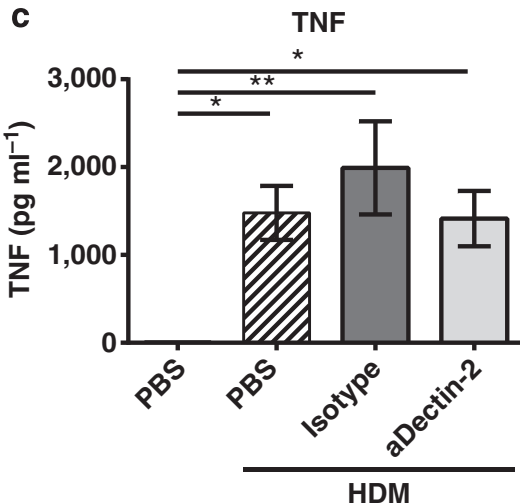

b

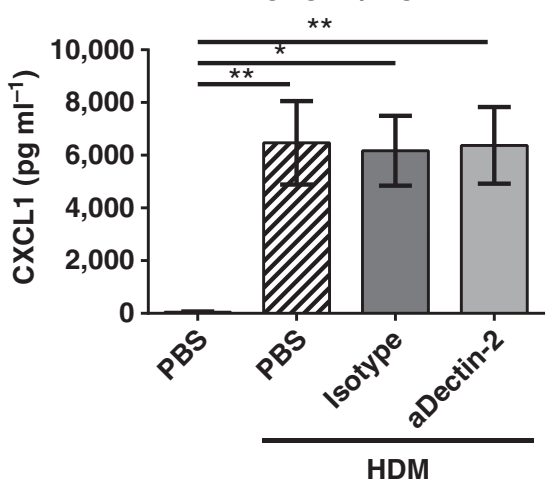

d

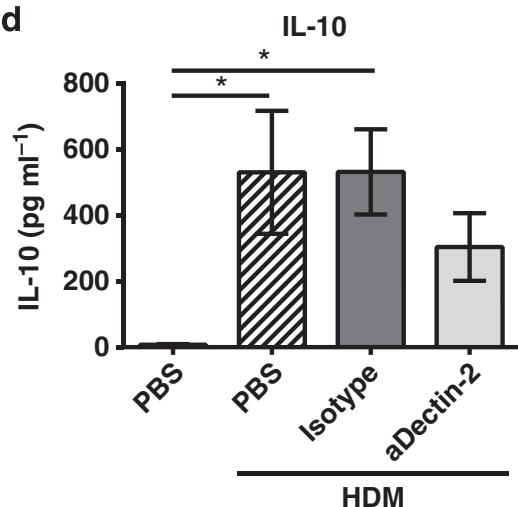

Figure 4 Dectin-2 regulates house dust mite (HDM)-induced cysteinyl leukotriene but not cytokine responses in primary murine alveolar macrophages. BALF cells from unchallenged mice (>95\% alveolar macrophages) were plated overnight in GM-CSF $\left(10 \mathrm{ng} \mathrm{ml}^{-1}\right)$, and then pre-treated with antibodies $\left(10 \mu \mathrm{g} \mathrm{ml}^{-1}\right) 1 \mathrm{~h}$ before HDM $\left(10 \mu \mathrm{g} \mathrm{ml}^{-1}\right)$ stimulation. Cysteinyl leukotriene generation was assessed at $30 \mathrm{~min}(\mathrm{a})$ and cytokine induction after $24 \mathrm{~h}(\mathbf{b}-\mathbf{d})$. Data are expressed as mean \pm s.e.m., $n=3$ performed in triplicate. Significance was determined by 1 -way analysis of variance comparing all groups. ${ }^{*} P<0.05,{ }^{* *} P<0.01$.

immunohistochemical analysis suggests that Dectin-2 is primarily expressed by alveolar macrophages, and not dendritic cells, in the lungs of naïve mice. ${ }^{9}$ We have also detected Dectin2 on alveolar macrophage by FACS analysis (data not shown). We were unable to selectively deplete alveolar macrophages without inducing a background inflammation making it challenging to directly demonstrate alveolar macrophages as the responding cell. Together these data indicate that Dectin-2 has a critical role in the triggering of HDM-induced inflammation, and this is mediated by alveolar macrophages.

Consistent with a central role in the induction of airway inflammation, alveolar macrophages produced CXCL1/KC, $\mathrm{TNF} \alpha$, and cysteinyl leukotrienes when stimulated ex vivo with HDM. Although Dectin-2 triggering can induce cytokines, including $\mathrm{TNF}^{8}{ }^{8}$ the blocking antibody had no effect on cytokines or chemokine indicating redundancy with another receptor such as TLR4. However, the induction of cysteinyl leukotrienes was Dectin-2 dependent both ex vivo and in vivo. Previously, reports show that pulmonary CD11c-expressing cells, but not alveolar macrophages release cysteinyl leukotriene in response to HDM. ${ }^{6}$ However, in this study by Barrett et al. ${ }^{6}$ the pulmonary dendritic cells, but not the alveolar macrophages, were primed with GM-CSF. Priming of alveolar macrophages with GM-CSF enhances cysteinyl leukotriene release (data not shown and Suram et al. ${ }^{11}$ ) and was required to see the Dectin-2 effect. GM-CSF is most highly expressed in the lungs of mice, ${ }^{12}$ and we have found low levels of GM-CSF in the BALF of naïve and challenged mice (data not shown). This is consistent with the requirement for GM-CSF for alveolar macrophage differentiation/function. ${ }^{13}$ Additionally, GM-CSF is reportedly induced by HDM in lung tissue and by epithelial cell cultures ${ }^{14}$ and is important in the response to acute and chronic HDM responses. ${ }^{10}$ Therefore, we propose GM-CSF priming of the alveolar macrophages merely reflects the physiological conditions in the lung, as evidenced by the very similar observations in vivo (Figure 3d).

In the single HDM challenge model, the leukotriene synthesis inhibitor zileuton inhibited neutrophil recruitment, but not chemokine/cytokine production, thus paralleling Dectin-2 blockade in vitro and in vivo. This indicates that Dectin-2 induction of cysteinyl leukotrienes could be the critical inflammatory trigger. Cysteinyl leukotrienes have been shown to have a number of different roles in allergic airway inflammation and can activate epithelial cells, endothelium, smooth muscle, and multiple leukocytes. ${ }^{15}$ It has long been established that leukotrienes are important for antigen recall in the lung, as demonstrated by the OVA model. ${ }^{16}$ However, it is only more recently that their importance in the HDM model 


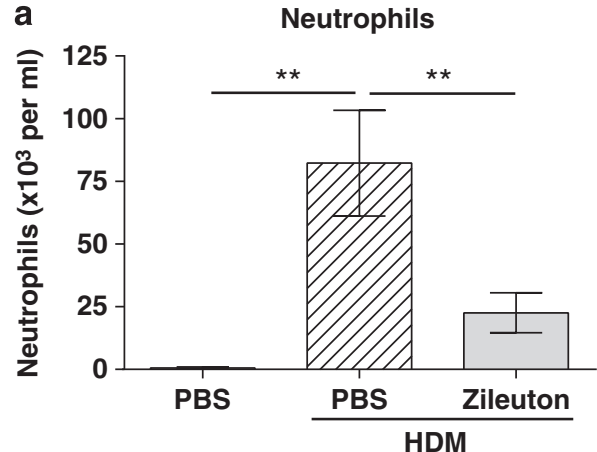

b
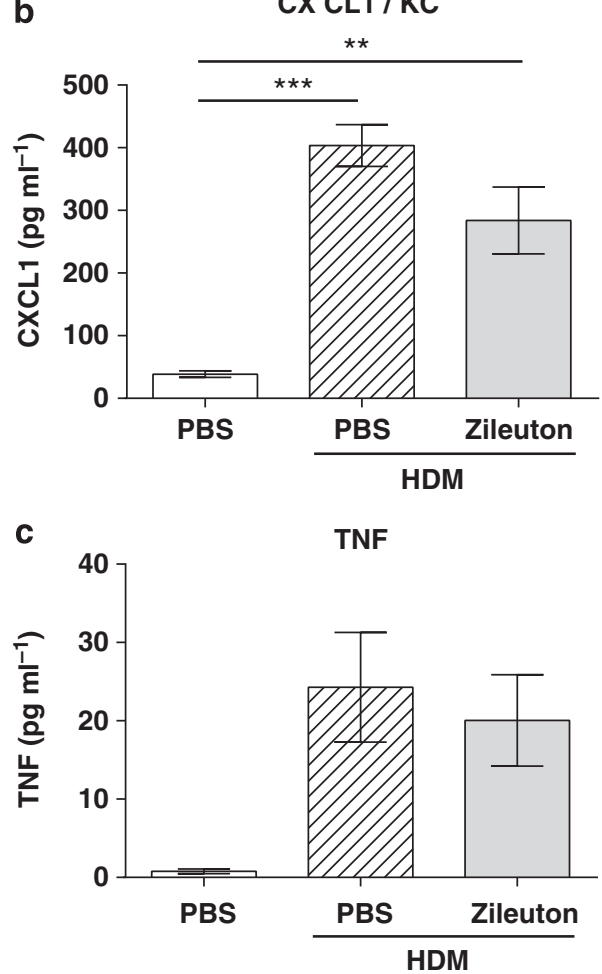

Figure 5 The synthesis of leukotrienes is required for the innate sensing of house dust mite (HDM). Mice were treated with the 5-lipoxygenase inhibitor zileuton $\left(10 \mathrm{mg} \mathrm{kg}^{-1}\right.$; p.o.) or vehicle $(0.5 \%$ methylcellulose containing $0.2 \%$ Tween 80 ; p.o.) $1 \mathrm{~h}$ before, 3 and $7 \mathrm{~h}$ post HDM challenge. Cellular recruitment to the airway lumen was determined by bronchoalveolar lavage, followed by differential counting (a). BALF was assayed for cytokine content by Meso-Scale Discovery (b, c). Data are expressed as mean \pm s.e.m., $n=6-8$ mice per group, and significance was determined by 1 -way analysis of variance comparing all groups. ${ }^{* \star} P<0.01,{ }^{* \star *} P<0.001$.

has been demonstrated by the lack of inflammation observed in the CysLTR1 knock out. ${ }^{17}$ This is supported by our observations with zileuton in the chronic HDM model. It is less clear how cysteinyl leukotrienes could lead to acute recruitment of neutrophils. This may not be direct but rather may be a permissive or indirect requirement. For example, cysteinyl leukotrienes are vasoactive and could be involved in the endothelial activation that is critical for neutrophil transmigration. ${ }^{18}$ This hypothesis is supported by the relatively small effect of anti-Dectin-2 on CXCL1/KC levels, a very potent neutrophil chemoattractant.
We therefore propose that the initial inflammatory response to HDM requires Dectin-2-triggered induction of cysteinyl leukotriene from alveolar macrophages. This represents a novel axis in allergen-driven airway inflammation. This is clearly distinct from, but parallels, the role for Dectin-2 expressed on dendritic cells in the priming of the Th2 response to HDM described by Barrett et al. ${ }^{7}$ Because of the continuing challenge/restimulation nature of the aeroallergens models, we cannot determine the relative contribution of the axes to the chronic aeroallergen-induced inflammation. Although the cell reconstitution experiments by Barrett et al. ${ }^{7}$ do indicate that induction of cysteinyl leukotrienes via dendritic cell expressed Dectin-2 is necessary for T-cell priming, which is consistent with our observed effects on the Th2 pathway. This does not exclude the possibility that the initaiation of inflammation by the alveolar macrophages does not impact on the adaptive response, for example, it could recruit DCs to the airway lumen or indirectly cause exposure of tissue DCs to the aeroallergen. It is interesting to note, that despite the impact on Th2 cytokines, we did not see an effect on serum IgE levels (data not shown). Clearly, Dectin-2 expression on alveolar macrophages and dendritic cells is important for the response to HDM, but it is worth noting Dectin-2 could potentially be expressed by other infiltrating cells, such as eosinophils. HDM-triggered PRRs are not just required by innate immune cells. For example, TLR4 expression on airway stromal cells is necessary for HDM-driven airway inflammation, but not on hematopoietic cells. ${ }^{5}$ This may again reflect redundancy on innate immune cells. It is clear that the involvement of multiple PRRs on multiple cells combine to cause a pathological response to the complex aeroallergen HDM.

PRRs discriminate foreign from host by recognizing structural features on microbes known as pathogen-associated molecular patterns (PAMPs), for example, TLR4 primarily recognizes the Gram-negative bacterial cell wall component, lipolysaccharide. ${ }^{19,20}$ LPS is present at varying levels in HDM extract, ${ }^{21}$ and commensal bacteria have been presumed to be the source, especially as fecal pellets can be present in extracts. ${ }^{22}$ This exogenous source of PAMP is thought to be physiologically relevant, because HDM fecal pellets themselves are allergenic and the HDM-contaminated dust will have contaminating microbes. Although in addition the HDM protein Der $\mathrm{p} 2$ is a mimetic of MD2, the LPS-binding co-receptor of TLR4, and may confer an increase in host sensitivity. ${ }^{23}$ The primary PAMP of Dectin-2 is thought to be high mannosylated structures expressed by fungi. ${ }^{24,25} \mathrm{HDM}$ are also known to carry fungi, ${ }^{26}$ therefore Dectin-2, like TLR4, could be triggered by HDMassociated microbes. Indeed, HDM may be a potent aeroallergen because of the combination of microbial products and HDMexpressed factors, such as Der p2 and cysteine proteases.

In the relatively small human biopsy arm of the study, Dectin-2 immunostaining was demonstrated in non-asthma controls and the asthma samples of GINA 1-4 stage with the number of Dectin-2-positive cells in the lamina propria being consistent between the sample sets. It is worth noting that, the total number of infiltrating cells was actually slightly decreased 

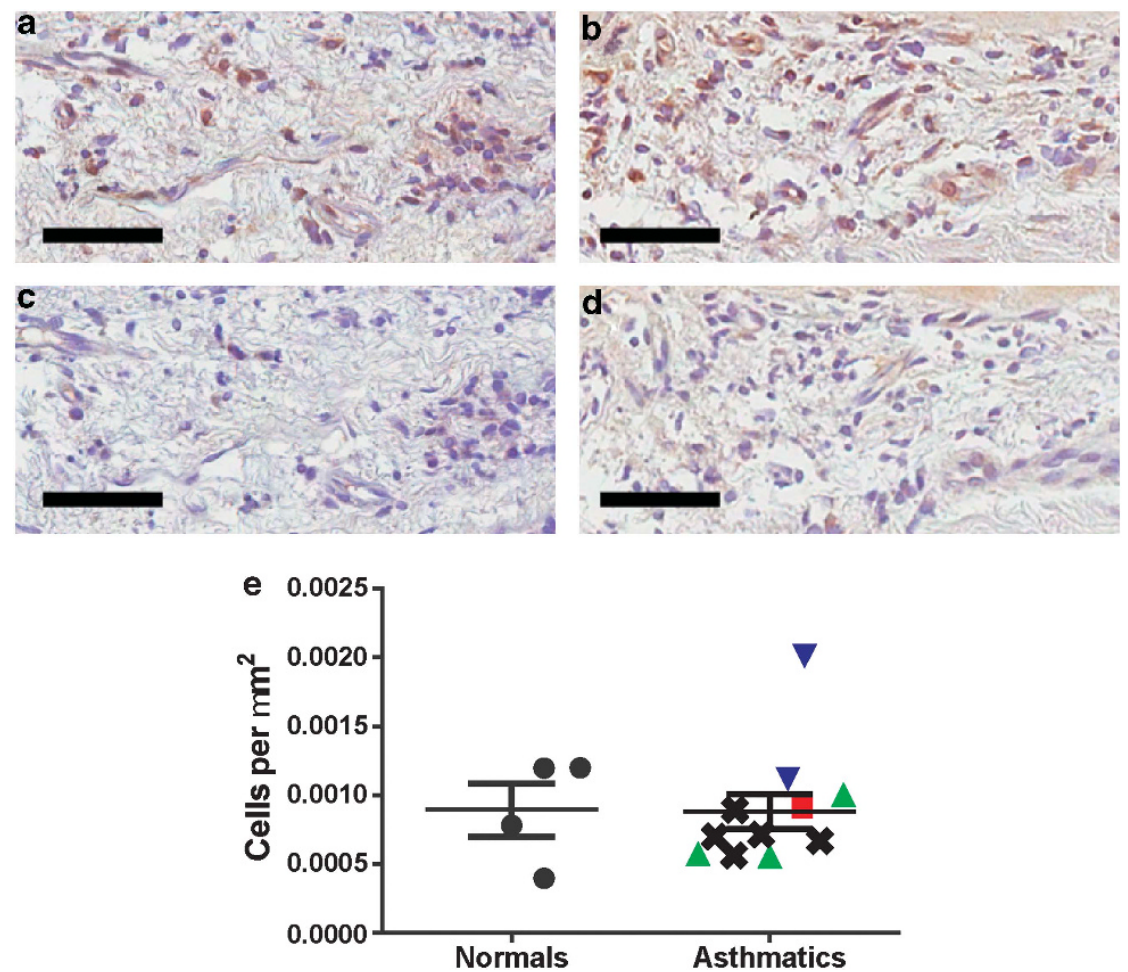

Figure 6 Dectin-2 expression in bronchial biopsies from non-smoking healthy and asthmatic patients. Anti-Dectin-2 (a,b) or rabbit IgG control (c,d) immunohisotchemical staining of brochial biopsies from a non-smoking healthy (a,c) or GINA 2 asthmatic patient (b,d). Typical areas of laminar propria are shown, and bar represents $50 \mu \mathrm{m}$. (e) Quantification of Dectin-2-positive cells per area of lamina propria in bronchial biopsies from healthy controls $(\bullet)$ or asthmatic patients (X GINA1; $\mathbf{G I N A 2 ;} \boldsymbol{\nabla}$ GINA3; $\Delta$ GINA4). Mean \pm s.e.m. are shown for healthy controls and total asthmatic patients. Open symbols represent quantification with control IgG.

in areas analyzed in the asthmatic biopsies compared with the control, and, as such, the percentage of infiltrating cells positive for Dectin-2 was similar or slightly increased (data not shown). It is possible that the anti-inflammatory medication of the GINA2-4 patients may have impacted significantly on the number of infiltrating cells, and consequently the number of Dectin-2-positive cells. Importantly, morphometric analysis located Dectin-2 expression to infiltrating mononuclear cells and macrophages. This would be consistent with Dectin-2 having a similar role in human allergic airway inflammation, as we observe in the mouse models.

In summary, our data show a requirement for Dectin-2 in alveolar macrophage induction of cysteinyl leukotrienes and in the initiation of airway inflammation. This information is critical for our understanding of the complex responses that lead to the aberrant inflammation triggered by aeroallergens. In addition, we elucidate an underappreciated role of cysteinyl leukotrienes in the initial response to the prototypical aeroallergen HDM. Finally, we showed expression of Dectin-2 in human lung.

\section{METHODS}

Mice. Female BALB/c mice (6-8 weeks) were purchased from Charles River Laboratories (Kent, UK) and housed at the MedImmune Animal facility. All work was carried out according to UK Home Office ethical and husbandry standards under the authority of an appropriate project licence.
Induction of airway inflammation. Chronic HDM model: Isofluorane-anaesthetized female BALB/c mice (Charles River Laboratories) were intranasally administered $25 \mu \mathrm{g}$ of $\mathrm{HDM}$ extract (total protein; Greer, Lenoir, NC) in $25 \mu$ l of phosphate-buffered saline (PBS) or PBS three times per week for 3 weeks ${ }^{27}$ and killed $24 \mathrm{~h}$ after the last challenge. $350 \mu$ g per mouse anti-Dectin-2 (D2.11E4; Antibody Serotec, Oxford, UK) or rat IgG2a isotype antibody (Biolegend, San Diego, CA) were administered intraperitoneally either 1 day before the first challenge and every third day thereafter (prophylactic) or every third day throughout the last 2 weeks (therapeutic). Zileuton $(5,15$, or $50 \mathrm{mg} \mathrm{kg}^{-1}$; Sigma-Aldrich, St Louis, MO) or vehicle $(0.5 \%$ methylcellulose $/ 0.2 \%$ Tween 80 in water) was dosed orally twice daily 1 day before and throughout the chronic model. Prednisone was dosed orally daily for the last 2 weeks $\left(10 \mathrm{mg} \mathrm{kg}^{-1}\right)$. AHR was determined by the FlexiVent (SCIREQ, Montreal, QC, Canada) in response to aerosolized methacholine (Sigma-Aldrich) following anesthetization with pentobarbital sodium.

Single-challenge model: Female $\mathrm{BALB} / \mathrm{c}$ mice were intranasally challenged once with HDM extract or PBS, as above, and killed $24 \mathrm{~h}$ later. Antibodies were administered as above $24 \mathrm{~h}$ before challenge. Zileuton $\left(10 \mathrm{mg} \mathrm{kg}^{-1}\right.$; Sigma-Aldrich) or vehicle (0.5\% methylcellulose $/ 0.2 \%$ Tween 80 in water) was dosed orally $1 \mathrm{~h}$ before, then $3 \mathrm{~h}$ and $7 \mathrm{~h}$ post, HDM challenge.

Airways were lavaged with PBS $(3 \times 0.3 \mathrm{ml})$, BALF centrifuged and cells counted. Differential cell counts (200 cells per slide) were performed on cytospin preparations stained with Diff-Quik (Fisher Scientific, Loughborough, UK).

Mouse histology. Mouse lung samples were inflated and fixed with $10 \%$ neutral-buffered formalin for $24 \mathrm{~h}$ and then processed and embedded into paraffin wax blocks. Sections were stained with hematoxylin and eosin ( $\mathrm{H} \& \mathrm{E})$ and scanned using the Aperio 
Scanscope XT. Each slide was assessed and scored manually according to the inflammation around the main airways using a semi-quantitative scoring system where $0=$ no change, $1=$ slight, $2=$ mild, $3=$ moderate, $4=$ marked and $5=$ severe.

Analysis of cytokines, chemokines and cysteinyl leukotrienes. Lung homogenate (10\% weight/volume in PBS) and BALF protein levels were measured using either enzyme-linked immunosorbent assays (ELISAs) (R\&D systems) or MSD (Meso-Scale Discovery; R\&D Systems). Levels were normalized to mean values from HDM/PBStreated animals for each independent experiment. Absolute levels of HDM-induced cytokine and chemokine levels are shown in Supplementary Tables S2, S3 and S4. Cysteinyl leukotriene levels were quantified in the BALF using the Luminex Kit (Cayman Chemical, Ann Arbor, MI). mRNA was purified using an RNeasy Plus mini kit (Qiagen, Hilden, Germany) and cDNA was synthesized using high-capacity RNA:cDNA kit (Applied Biosystems, Grand Island, NY). Gene expression was measured using custom-designed low-density array cards or inventories primer and probes sets (RANTES/CCL5 and TGF $\beta$; Applied Biosystems) and normalized to $18 \mathrm{~S}$ RNA, and expressed as fold change over PBS alone treatment. The proteins and transcripts analyzed are listed in Supplementary Table S1.

Culture of alveolar macrophages. BALF cells were plated (20 000 cells per well) in RPMI containing 10\% FBS, penicillin/streptomycin (Sigma-Aldrich) and $10 \mathrm{ng} \mathrm{ml}^{-1}$ GM-CSF (R\&D Systems, Minneapolis, MN) and cultured overnight. Cells were pre-treated with antiDectin-2 or isotype control $\left(10 \mu \mathrm{g} \mathrm{ml}^{-1}\right)$ for $1 \mathrm{~h}$ before stimulation with $10 \mu \mathrm{g} \mathrm{ml}^{-1} \mathrm{HDM}$ extract. Cysteinyl leukotriene levels were quantified using the Luminex kit (Cayman Chemical). mRNA levels, cytokine and chemokines were measured as above.

Human subjects and tissue immunohistochemistry. Human asthma bronchial biopsy samples were obtained with written informed consent at the Wythenshaw Hospital, Manchester. The demographics of the asthmatic patients are shown in Supplementary Table S2. Tissue was fixed for $48 \mathrm{~h}$ in $10 \%$ neutral-buffered formalin within $2-3 \mathrm{~h}$ of surgery before embedding in.paraffin. Sections were stained using a rabbit anti-human Dectin-2 antibody (Ab68998, $50 \mu \mathrm{g} \mathrm{ml}^{-1}$, Abcam, Cambridge, MA) or Rabbit IgG (Dako, Carpinteria, CA) as a control and counterstained in Gills II Haematoxylin.

The slides were scanned using a Scanscope XT (Aperio). Using a digital tablet display (Cintiq 21UX; Wacom), the images were manually annotated to select the area(s) of lamina propria and to exclude blood vessel structures. An image analysis algorithm (IHC Nuclear V1; Aperio) was modified to select all stained cells present. All samples, where a suitable image was available, were analyzed.

Statistical analysis. Results are expressed as mean \pm s.e.m., analyzed by one-way analysis of variance followed by Bonferroni post-test comparing all groups, except: Figures 1a, Supplementary Figures S1B and $\mathbf{2 A}$ were analyzed by two-way analysis of variance with methacholine dose used as the block; and Figure 1e expressed as median with range. Graph generation and statistical analyses were performed using Prism (GraphPad).

SUPPLEMENTARY MATERIAL is linked to the online version of the paper at http://www.nature.com/mi

\section{ACKNOWLEDGMENTS}

We thank Elaine Wilson and Sarah Bolton for their technical assistance with the immunohistochemical studies. We are also indebted to Athula Herath for assistance with the statistical analysis, and Raffaella Faggioni for advice on the antibody-dosing regimen. Jayesh Majithiya, Tamara Baker and Helen Brant were of considerable assistance for all the in vivo studies. This work was supported by Medlmmune Ltd.

\section{DISCLOSURE}

All contributors were employed by Medlmmune Ltd or AstraZeneca during these studies.

(c) 2014 Society for Mucosal Immunology

\section{REFERENCES}

1. Craig, T.J. Aeroallergen sensitization in asthma: prevalence and correlation with severity. Allergy Asthma Proc 31, 96-102 (2010).

2. Janeway, C.A. Jr. Approaching the asymptote? Evolution and revolution in immunology. Cold Spring Harb Symp Quant Biol 54, 1-13 (1989).

3. Svensson, L. et al. House dust mite allergen activates human eosinophils via formyl peptide receptor and formyl peptide receptor-like 1. Eur $J$ Immunol 37, 1966-1977 (2007).

4. Asokananthan, N. et al. House dust mite allergens induce proinflammatory cytokines from respiratory epithelial cells: the cysteine protease allergen, Der p 1, activates protease-activated receptor (PAR)-2 and inactivates PAR-1. J Immunol 169, 4572-4578 (2002).

5. Hammad, H. et al. House dust mite allergen induces asthma via Toll-like receptor 4 triggering of airway structural cells. Nat Med 15, 410-416 (2009).

6. Barrett, N.A., Maekawa, A, Rahman, O.M., Austen, K.F. \& Kanaoka, Y Dectin-2 recognition of house dust mite triggers cysteinyl leukotriene generation by dendritic cells. J Immunol 182, 1119-1128 (2009).

7. Barrett, N.A. et al. Dectin-2 mediates Th2 immunity through the generation of cysteinyl leukotrienes. J Exp Med 208, 593-604 (2011).

8. Robinson, M.J. et al. Dectin-2 is a Syk-coupled pattern recognition receptor crucial for Th17 responses to fungal infection. J Exp Med 206, 2037-2051 (2009).

9. Taylor, P.R. et al. Dectin-2 is predominantly myeloid restricted and exhibits unique activation-dependent expression on maturing inflammatory monocytes elicited in vivo. Eur J Immunol 35, 2163-2174 (2005).

10. Cates, E.C. et al. Intranasal exposure of mice to house dust mite elicits allergic airway inflammation via a GM-CSF-mediated mechanism. J Immunol 173, 6384-6392 (2004).

11. Suram, S. et al. Pathways regulating cytosolic phospholipase A2 activation and eicosanoid production in macrophages by Candida albicans. J Biol Chem 285, 30676-30685 (2010).

12. Willinger, T. et al. Human IL-3/GM-CSF knock-in mice support human alveolar macrophage development and human immune responses in the lung. Proc Natl Acad Sci USA 108, 2390-2395 (2011).

13. Trapnell, B.C., Carey, B.C., Uchida, K \& Suzuki, T. Pulmonary alveolar proteinosis, a primary immunodeficiency of impaired GM-CSF stimulation of macrophages. Curr Opin Immunol 21, 514-521 (2009).

14. Willart, M. et al. Interleukin-1alpha controls allergic sensitization to inhaled house dust mite via the epithelial release of GM-CSF and IL-33. J Exp Med 209, 1505-1517 (2012).

15. Peters-Golden, M. Expanding roles for leukotrienes in airway inflammation. Curr Allergy Asthma Rep 8, 367-373 (2008).

16. Austen, K.F. Additional functions for the cysteinyl leukotrienes recognized through studies of inflammatory processes in null strains. Prostaglandins Other Lipid Mediators 83, 182-187 (2007).

17. Maekawa, A, Xing, W, Austen, K.F. \& Kanaoka, Y. GPR17 regulates immune pulmonary inflammation induced by house dust mites. $J$ Immunol 185, 1846-1854 (2010).

18. Sala, A \& Folco, G. Neutrophils, endothelial cells, and cysteinyl leukotrienes: a new approach to neutrophil-dependent inflammation? Biochem Biophys Res Commun 283, 1003-1006 (2001).

19. Medzhitov, R, Preston-Hurlburt, P \& Janeway, C.A. Jr. A human homologue of the Drosophila Toll protein signals activation of adaptive immunity. Nature 388, 394-397 (1997).

20. Poltorak, A. et al. Defective LPS signaling in $\mathrm{C} 3 \mathrm{H} / \mathrm{HeJ}$ and C57BL/10ScCr mice: mutations in TIr4 gene. Science (New York, N.Y 282, 2085-2088 (1998).

21. Post, S. et al. The composition of house dust mite is critical for mucosal barrier dysfunction and allergic sensitisation. Thorax 67, 488495 (2012).

22. Gregory, L.G. \& Lloyd, C.M. Orchestrating house dust mite-associated allergy in the lung. Trends in immunology 32, 402-411 (2011). 
23. Trompette, A. et al. Allergenicity resulting from functional mimicry of a Toll-like receptor complex protein. Nature 457, 585-588 (2009).

24. McGreal, E.P. et al. The carbohydrate-recognition domain of Dectin-2 is a C-type lectin with specificity for high mannose. Glycobiology 16, 422-430 (2006).

25. Sato, K. et al. Dectin-2 is a pattern recognition receptor for fungi that couples with the Fc receptor gamma chain to induce innate immune responses. J Biol Chem 281, 38854-38866 (2006).

26. Hay, D.B., Hart, B.J., Pearce, R.B., Kozakiewicz, Z \& Douglas, A.E. How relevant are house dust mite-fungal interactions in laboratory culture to the natural dust system? Exp Appl Acarol 16, 37-47 (1992).
27. Gregory, L.G. et al. Inhaled house dust mite induces pulmonary $\mathrm{T}$ helper 2 cytokine production. Clin Exp Allergy 39, 15971610 (2009).

(c) (1) () () This work is licensed under the Creative Commons Attribution-NonCommercial-No

Derivative

Works 3.0 Unported License. To view a copy of this license, visit http://creativecommons.org/licenses/by-nc-nd/3.0/ 